\title{
Autophagy down-regulates NLRP3-dependent inflammatory response of intestinal epithelial cells under nutrient deprivation
}

\author{
Yewon Yun, Ahruem Baek \& Dong-Eun Kim ${ }^{*}$ \\ Department of Bioscience and Biotechnology, Konkuk University, Seoul 05029, Korea
}

\begin{abstract}
Dysregulation of inflammation induced by noninfectious stress conditions, such as nutrient deprivation, causes tissue damage and intestinal permeability, resulting in the development of inflammatory bowel diseases. We studied the effect of autophagy on cytokine secretion related to intestinal permeability under nutrient deprivation. Autophagy removes NLRP3 inflammasomes via ubiquitin-mediated degradation under starvation. When autophagy was inhibited, starvation-induced NLRP3 inflammasomes and their product, IL-1 $\beta$, were significantly enhanced. A prolonged nutrient deprivation resulted in an increased epithelial mesenchymal transition (EMT), leading to intestinal permeability. Under nutrient deprivation, IL-17E/25, which is secreted by IL-1 $\beta$, demolished the intestinal epithelial barrier. Our results suggest that an upregulation of autophagy maintains the intestinal barrier by suppressing the activation of NLRP3 inflammasomes and the release of their products, including proinflammatory cytokines IL-1 $\beta$ and IL-17E/25, under nutrient deprivation. [BMB Reports 2021; 54(5): 260-265]
\end{abstract}

\section{INTRODUCTION}

Inflammatory bowel disease (IBD) is a chronic inflammatory disease of the gastrointestinal tract (GI). Gl has epithelial tight junction barriers, composed of a series of individual cells that prevent luminal contents, such as pathogenic organisms and gastric acid, from leaking (1). Intestinal epithelial tight junctions are important components in the intestinal defense system (2). This intestinal barrier can be disrupted by an excessive inflammatory and immune response against infection by pathogens or various stress conditions (3). Consequently, defective intestinal epithelial barriers cause tissue damage with enhanced intestinal permeability, causing inflammatory bowel diseases, such as

*Corresponding author. Tel: +82-2-2049-6062; Fax: +82-2-3436-6062; E-mail: kimde@konkuk.ac.kr

https://doi.org/10.5483/BMBRep.2021.54.5.211

Received 25 September 2020, Revised 30 October 2020, Accepted 11 December 2020

Keywords: Autophagy, Cytokine, Epithelial mesenchymal transition, NLRP3 inflammasome, Nutrient deprivation
Crohn's disease (CD) (4), ulcerative colitis (UC) (5), and leaky gut syndrome (6).

NLRP3 (nod-like receptor family pyrin domain containing 3 ) inflammasomes, which are multiprotein complexes composed of NLRP3, ASC (the adaptor molecule apoptosis associated specklike protein containing a CARD), and pro-Caspase-1, are critical components of the inflammatory response to maintain intestinal homeostasis (7). NLRP3 inflammasomes that are activated by various danger signals of pathogenic and nonpathogenic sources produces the pro-inflammatory cytokines, such as interleukin- $1 \beta$ (IL-1 $\beta$ ) and IL-18 (8). However, NLRP3 inflammasome dysregulation has been known to cause several chronic inflammatory, infectious, and autoimmune diseases (9). Since pro-inflammatory cytokines can modulate epithelial barrier function, an uncontrolled activation of NLRP3 inflammasomes elevates intestinal permeability, leading to intestinal epithelium destruction (10).

Autophagy, a cellular process of lysosomal degradation, is a cellular stress response that plays an essential roles in physiological processes, such as the removal of damaged organelles (11), antimicrobial defense (12), protein secretion (13), and inflammatory response (14). Autophagy is a negative regulator of NLRP3 inflammasomes during an inflammatory response (15). Regarding the anti-inflammatory effect of autophagy, autophagy impairment enhances the accumulation of inflammasomes (16). Moreover, autophagy dysregulation leads to the accumulation of various toxic macromolecules, including damaged organelles and oxidized proteins (17). Thus, it has been suggested that autophagy dysregulation, which is a common IBD feature, causes an abnormal inflammasome activity (18). However, to date, the molecular mechanisms underlying the sequential correlation between NLRP3 inflammasomes and autophagy in intestinal epithelial cells have not been fully delineated.

Herein, we report that autophagy dysregulation is a possible cause of intestinal epithelial barrier disruption under nutrient deprivation. We investigated whether NLRP3 inflammasomes were significantly activated when autophagy was inhibited by monitoring NLRP3 inflammasome levels. We monitored whether the cytokines secreted due to NLRP3 inflammasome activation promote intestinal permeability. NLRP3 inflammasome was activated during short-term exposure to starvation but was attenuated during the induction of the autophagic process during long-term exposure to starvation. 


\section{RESULTS AND DISCUSSION}

\section{Oxidative stress induces the formation of NLRP3 inflammasomes and autophagic flux under nutrient deprivation}

We first examined the generation of reactive oxygen species (ROS) in Caco-2 cells that were subjected to nutrient deprivation by monitoring cellular fluorescence (Fig. 1A). ROS fluorescence was increased in a starvation-time-dependent manner. Since ROS generation is known to induce inflammation, we next investigated whether the ROS generated by starvation induce NLRP3 inflammasomes in cells. In nutrient-deprived cells, the accumulation of NLRP3 inflammasome components, such as NLRP3, ASC, pro-Caspase-1, and cleaved-Caspase-1, was observed after $6 \mathrm{~h}$, whereas the levels of NLRP3 inflammasome components gradually decreased over time (Fig. 1B). To verify the formation of NLRP3 inflammasomes during starvation, the presence of NLRP3 and ASC in Caco-2 cells under nutrient deprivation were traced using green and red florescence, respectively (Fig. 1C). The fluorescence of NLRP3 and ASC was colocalized in nutrient-deprived cells (for $6 \mathrm{~h}$ ), while the colocalization of NLRP3 and ASC was significantly diminished when the cells were exposed to starvation for a long period (after $6 \mathrm{~h}$ ).

Next, we investigated whether nutrient deprivation induces autophagy in Caco-2 cells. When the cells were exposed to nutrient deprivation, sequestosome 1 (SQSTM1) level was decreased and the ratio of LC3B-I to LC3B-II conversion was increased for a long period (after $12 \mathrm{~h}$ ) (Fig. 1D). This result indicates that NLRP3 inflammasome formation and autophagy induction are early and late events, respectively, in Caco-2 cells under nutrient deprivation. We also investigated the autophagic flux in Caco-2 cells under nutrient deprivation by monitoring the autophagosome formation and autophagosome-lysosome fusion. Autophagosomes and lysosomes were traced using GFP-LC3B and a lysosomespecific fluorescent dye (LysoTracker Red DND-99), respectively (Fig. 1E). GFP-LC3B puncta were significantly increased in nutrient-deprived cells, compared to normal cells. In the merged image, the up-regulated GFP-LC3B puncta due to starvation colocalized with red-colored lysosomes. Thus, the autophagic flux was gradually enhanced in Caco-2 cells under prolonged nutrient deprivation.

\section{Autophagy down-regulates NLRP3 inflammasomes under nutrient deprivation}

Based on the result that the early elevated NLRP3 inflammasomes under starvation gradually decreased over time, we hypothesized that the activation of NLRP3 inflammasomes in the early stages of starvation would decrease due to autophagy. It has been previously reported that NLRP3 inflammasomes can be degraded via autophagy (19). Thus, we examined whether autophagy can modulate the triggering of NLRP3 inflammasome with autophagic regulators in Caco-2 cells under nutrient deprivation. Bafilomycin A1 (Baf A1) was used to inhibit the autophagic flux by blocking the acidification of lysosomes. When
A

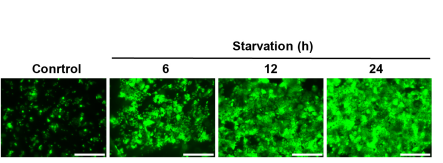
IIII B

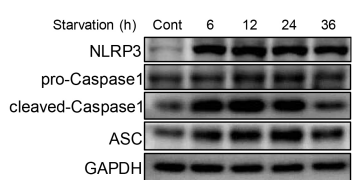

C
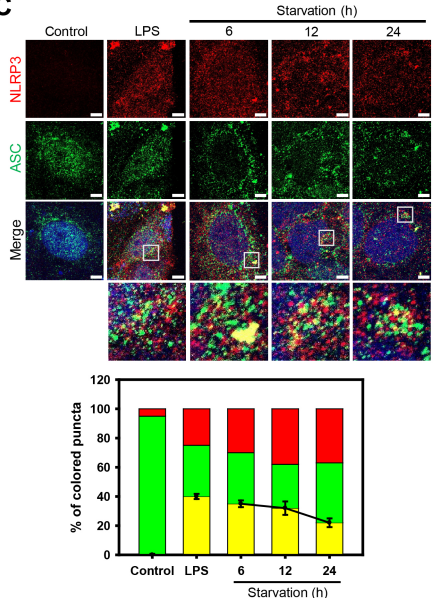

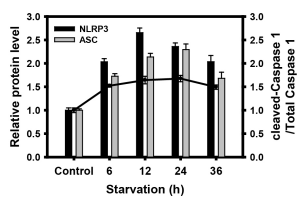

D



E

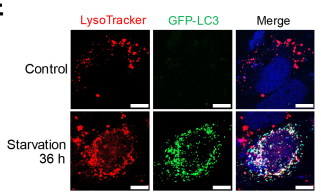

Fig. 1. Oxidative stress induces the formation of NLRP3 inflammasomes and autophagic flux under nutrient deprivation. (A) Fluorescence microscopy images of DCFDA-stained Caco-2 cells under nutrient deprivation for 6,12 , and $24 \mathrm{~h}$. Scale bar: $275 \mu \mathrm{m}$. Bar graph indicates the fluorescence intensity of DCF, which was normalized to control. The data are presented as the mean $\pm S D, n=3$. (B) Western blot analysis of NLRP3, pro-Caspase-1, cleaved-Caspase-1, and ASC in Caco-2 cells under nutrient deprivation for $6,12,24$, and 36 h. Bar graph indicates the levels of NLRP3 and ASC in the western blot analysis images. The protein band intensities were normalized to GAPDH. Dots in the graph indicates the ratio of cleaved-Caspase 1 to total Caspase 1 (pro-Caspase 1 plus cleaved-Caspase 1). Data are presented as the mean + SD of 3 independent experiments. (C) Fluorescence microscopy images of NLRP3 (red fluorescence) and ASC (green fluorescence) in Caco-2 cells under nutrient deprivation for 6,12 , and $24 \mathrm{~h}$ and treated lipopolysaccharides $(10 \mu \mathrm{g} / \mathrm{ml})$ for positive control of induced NLRP3 inflammasome. Scale bar: $5 \mu \mathrm{m}$. The fluorescent puncta of NLRP3 and ASC in the merged images were quantified and presented as a graph (red; puncta of NLRP3, green: puncta of ASC, yellow: co-localized puncta with NLRP3 and ASC); counts are the mean $\pm \mathrm{SD}$ of 3 independent experiments. (D) Western blot analysis of SOSTM1 and LC3B under nutrient deprivation for 6 , 12,24 , and $36 \mathrm{~h}$. The graph indicates the ratio of LC3B-II to LC3B-I in Fig. 1D and the levels of NLRP3 in Fig. 1B. The protein band intensities were normalized to GAPDH. Data are presented as the mean $\pm S D, n=3$. (E) Fluorescence microscopy images of Caco-2 cells were obtained by monitoring GFP-LC3 puncta (green fluorescence) and by staining them with LysoTracker Red DND-99 (red fluorescence) under nutrient deprivation for $36 \mathrm{~h}$. Scale bar: $10 \mu \mathrm{m}$. 
autophagy was inhibited by Baf A1, the secretion of IL- $1 \beta$ and Caspase-1, which represents the activity of NLRP3 inflammasomes, was significantly increased (Fig. 2A). In contrast, the autophagy inducer (torin 1)-treated groups showed little increase in the secreted levels of IL-1 $\beta$ and Caspase-1 under nutrient deprivation. Moreover, to further investigate whether the inhibition of autophagy initiation upregulates the activation of NLRP3 inflammasomes, we monitored the activity of IL-1 $\beta$ in ATG5 (autophagy-related 5)-knockdown cells (Fig. 2B). When the initiation of autophagy was inhibited by the knockdown of ATG5, the activity of IL-1 $\beta$ was significantly increased in Baf A1-treated cells.

We next investigated the protein expression level of the NLRP3 inflammasome in regulated autophagy condition under prolonged nutrient deprivation. When autophagy was inhibited by Baf A1 in Caco-2 cells under nutrient deprivation, the levels of NLRP3 and cleavage of Caspase-1were significantly increased compared to the group exposed to starvation without Baf A1 (Fig. 2C). In contrast, when autophagy was induced by torin 1 , the levels of NLRP3 and cleavage of Caspase- 1 were readily diminished over time under nutrient deprivation (Fig. 2D). Next, we examined whether autophagy affects the formation of NLRP3 inflammasomes by monitoring the components of the NLRP3 inflammasome in Caco-2 cells exposed to nutrient deprivation for a short period (6 h) with autophagic regulators (Fig. 2E). When the cells were exposed to nutrient deprivation stress, the levels of both NLRP3 and ASC were significantly increased, and ASC was translocated to the cytoplasm. The fluorescence of these proteins was colocalized in the cytoplasm with merged fluorescence (indicated as yellow fluorescence), reflecting the formation of NLRP3 inflammasomes. However, the autophagic inducer torin 1 decreased the level of NLRP3 inflammasome components (NLRP3 and ASC), as well as its colocalization, which was indicated by a diminished merged fluorescence. In contrast, Baf A1 treatment elevated the formation of NLRP3 inflammasomes under nutrient deprivation. Taken together, these results indicate that autophagy down-regulates NLRP3 inflammasomes in Caco-2 cells under nutrient deprivation.

It has been previously reported that NLRP3 inflammasomes are ubiquitinated and degraded through MARCH7/Axotrophin (20) and TRAF6 (21), the E3 ubiquitin ligases (22). These E3 ubiquitin ligases are known to be degraded by inflammasomes by autophagy after these are ubiquitinated (23). To address whether autophagy down-regulates NLRP3 inflammasomes through the degradation of these E3 ubiquitin ligases, we examined the levels of MARCH7/Axotrophin and TRAF6 in Caco-2 cells under nutrient deprivation. When the cells were exposed to starvation, MARCH7/Axotrophin and TRAF6 gradually decreased in a time-dependent manner, showing a similar pattern of NLRP3 inflammasome activation (Fig. 2F). However, the cells treated with autophagic inhibitors, such as Baf $\mathrm{A} 1$ and chloroquine (CQ), showed an accumulation of MARCH7/Axotrophin and TRAF6, compared to the untreated or torin 1-treated group under
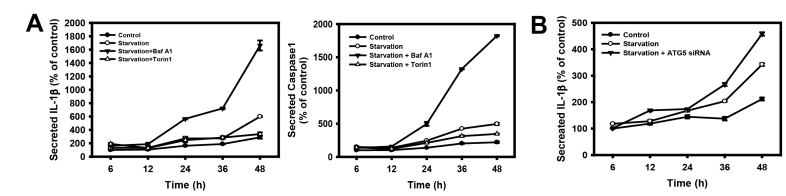

C
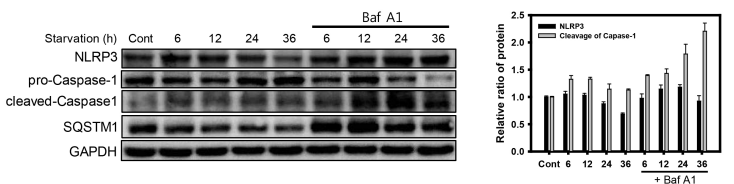

D
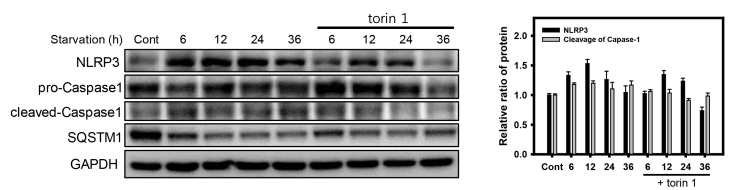

E

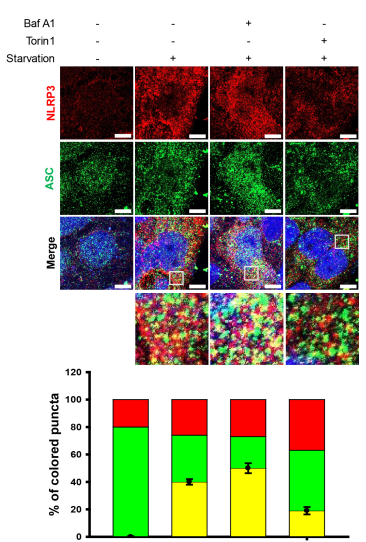

$\mathbf{F}$

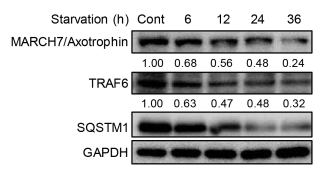

G



Fig. 2. Autophagy down-regulates NLRP3 inflammasomes under nutrient deprivation. (A) ELISA assay of IL-1 $\beta$ and Caspase-1 activity from cultured media in Caco-2 cells was treated with Baf A1 (20 nM) and torin $1(2 \mu \mathrm{M})$ under nutrient deprivation for $6,12,24,36$, and $48 \mathrm{~h}$. Activity was normalized to cell viability. (B) ELISA assay of IL-1 $\beta$ from cultured media in ATG5-knockdown Caco-2 cells under nutrient deprivation for $6,12,24,36$, and 48 h. Activity was normalized to cell viability. (C) Western blot analysis of NLRP3, pro-Caspase-1, and cleaved-Caspase-1 in Caco-2 cells under nutrient deprivation with or without Baf A1 $(20 \mathrm{nM})$. Bar graph indicates the levels of NLRP3, ASC, and SQSTM1 in the western blot analysis images. Protein band intensities were normalized to GAPDH. Data are presented as the mean \pm SD of 3 independent experiments. (D) Western blot analysis of NLRP3, pro-Caspase-1, and cleaved-Caspase-1 in Caco-2 cells under nutrient deprivation with or without torin $1(2 \mu \mathrm{M})$. Bar graph indicates the levels of NLRP3, ASC, and SQSTM1 in the western blot analysis images. The protein band intensities were normalized to GAPDH. Data are presented as the mean \pm SD of 3 independent experiments. (E) Fluorescence microscopy images of NLRP3 (red fluorescence) and ASC (green fluorescence) in Baf A1 $(20 \mathrm{nM})$, torin $1(2 \mu \mathrm{M})$, and LPS $(10 \mu \mathrm{g} / \mathrm{ml})$ in Caco-2 cells under nutrient deprivation for $6 \mathrm{~h}$. Scale bar: $5 \mu \mathrm{m}$. The fluorescent puncta of NLRP3 and ASC in the merged images were quantified and presented as a graph (red; puncta of NLRP3, green; puncta of ASC, yellow; co-localized puncta with NLRP3 and ASC); counts are the mean \pm SD of 3 independent experiments. (F) Western blot analysis of TRAF6, MARCH7/Axotrophin, and SQSTM1 in Caco-2 cells under nutrient deprivation for $6,12,24$, and $36 \mathrm{~h}$. (G) Western blot analysis of TRAF6, MARCH7/Axotrophin, and SQSTM1 in Baf A1 $(20 \mathrm{nM}), \mathrm{CQ}(50 \mu \mathrm{M})$, and torin $1(2 \mu \mathrm{M})$ in Caco-2 cells under nutrient deprivation for $36 \mathrm{~h}$. 
nutrient deprivation (Fig. 2G). These results indicate that NLRP3 inflammasomes are likely disposed of by autophagy through the degradation of E3 ubiquitin ligases during a long-term exposure to starvation.

\section{Prolonged nutrient deprivation increases permeability of intestinal epithelial cells}

Activation of NLRP3 inflammasomes and the secretion of cytokines by inflammasomes are known to induce intestinal permeability by disrupting the epithelial barrier (24-26). E-cadherin is an important junctional protein in epithelial cells that maintain the epithelial barrier (27). Thus, we examined whether autophagy affects the epithelial barrier function under nutrient deprivation by monitoring the expression of E-cadherin and vimentin, which are markers of epithelial-mesenchymal transition (EMT) (Fig. 3A). Under normal conditions, the cells maintained their epithelial morphology with a high expression of E-cadherin in the cell-cell junction and a low expression level of vimentin. Upon nutrient deprivation, the level of E-cadherin and vimentin was diminished and increased, respectively, indicating a progression of EMT. However, the inhibition of autophagy with Baf A1 or CQ significantly enhanced starvation-induced EMT, while the autophagy inducer (torin 1) slightly suppressed EMT under nutrient deprivation.

To further investigate the effect of autophagy on intestinal

A
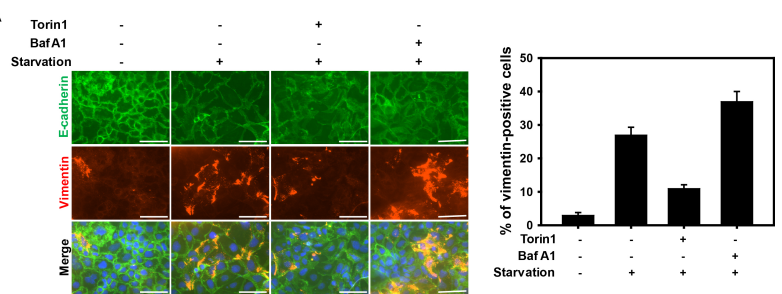

B


Fig. 3. Prolonged nutrient deprivation increases permeability of intestinal epithelial cells. (A) Caco-2 cells were treated with torin $1(2 \mu \mathrm{M})$ and Baf A1 (20 nM) under nutrient deprivation for $36 \mathrm{~h}$, and subsequently immunofluorescent staining of E-cadherin (green fluorescence) and Vimentin (red fluorescence). The nuclei were visualized with DAPI (blue fluorescence). Scale bar: $75 \mu \mathrm{m}$. The number of vimentinpositive cells were quantified and presented as a graph (vimentinpositive cells per total cells); counts are the mean \pm SD of 3 independent experiments. (B) Cell permeability assay of Caco-2 monolayers incubated in starvation for $36 \mathrm{~h}$ using a transwell. After incubation, cells were treated with FITC-Dextran $(100 \mu \mathrm{g} / \mathrm{ml})$ for $9 \mathrm{~h}$, and it was measured every hour. Data are presented as the mean \pm SD of 3 independent experiments. permeability under nutrient deprivation, we monitored the leakage of the intestinal epithelial layer by detecting the released fluorescence-labeled dextran (FITC-dextran) moving across the intestinal barrier (Fig. 3B). The cells exposed to starvation for $36 \mathrm{~h}$ showed increased levels of FITC-dextran, compared to normal cells (i.e., control cells). When the cells were treated with Baf A1 under nutrient deprivation conditions, the level of FITC-dextran was significantly increased. Similar to previous results (shown in Fig. 3A), the cells treated with torin 1 showed a diminished cell permeability under starvation conditions. Together, these results indicate that a prolonged nutrient deprivation promotes intestinal permeability by disrupting the epithelial barrier, and the inhibition of autophagy elevates starvation-induced intestinal permeability.

\section{IL-17E/25 promotes intestinal epithelial permeability under nutrient deprivation}

Intestinal epithelial cells are known to secrete cytokines in response to various stress conditions. To investigate the cytokines that enhance intestinal permeability under nutrient deprivation, we profiled the secreted cytokines from Caco-2 cells treated with autophagic regulators (Supplementary Fig. 1). IL-17E/25 was selected as a candidate that regulates intestinal permeability under starvation, due to the secretion pattern that depends on autophagy regulators. IL-17E/25 is known to promote Th2 cytokine production in response to allergens and parasitic infections (28), yet its function under other stress conditions is still poorly understood. To clarify whether the secretion of IL-17E/25 can be regulated by autophagy under nutrient deprivation, we monitored the secreted IL-17E/25 in Caco-2 cells in the absence or presence of autophagic regulators (Fig. 4A). Consistent with previous results (Fig. 2A), the level of secreted IL-17E/25 was increased in nutrient-deprived cells in a time-dependent manner. However, the inhibition of autophagy by Baf A1 significantly enhanced the secretion of IL-17E/25, which was induced by starvation. In contrast, the treatment with the autophagy inducer torin 1 significantly suppressed the level of secreted IL-17E/25 under a prolonged nutrient deprivation. This result suggests that the secretion of IL-17E/25 was down-regulated by autophagy, similar to the result regarding the secretion of IL-1 $\beta$ (Fig. $2 \mathrm{~A}$ ).

Next, we speculated whether the secretion of IL-17E/25 is promoted by IL-1 $\beta$, which mediates the inflammatory response for the activation and secretion of other cytokines under nutrient deprivation. We measured the level of secreted IL-17E/25 in Caco-2 cells exposed to recombinant human IL-1 $\beta$ (rhIL-1 $\beta$ ). The level of secreted IL-17E/25 was significantly increased by adding rhIL-1 $\beta$ in a dose-dependent manner (Fig. 4B). However, treatment with recombinant human IL-17E/25 (rhIL-17E/25) did not affect the level of secreted IL-1 $\beta$ (data not shown). These results indicate that nutrient deprivation induces IL-1 $\beta$-mediated IL-17E/25 secretion, which is likely down-regulated by autophagy. To further corroborate whether IL-17E/25 induces EMT, we monitored the expression of EMT markers in Caco-2 cells exposed to recombinant human IL-1 $\beta$ or IL-17E/25 (Fig. 4C). 
A

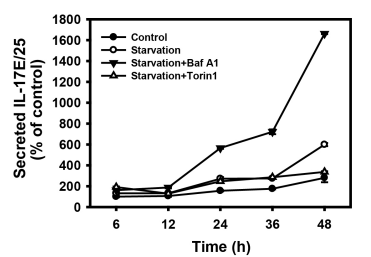

C

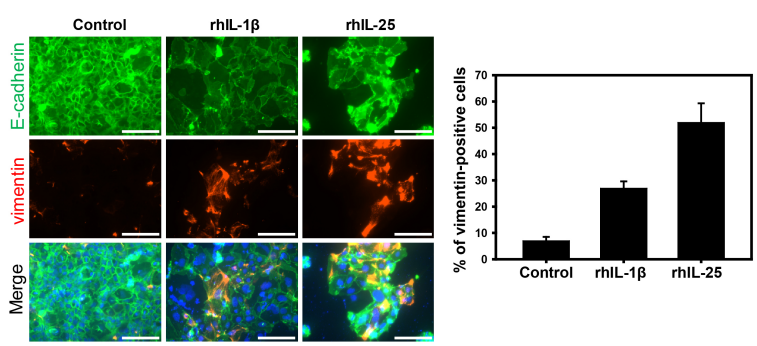

Fig. 4. IL-17E/25 promotes intestinal epithelial permeability under nutrient deprivation. (A) ELISA assay of IL-17E/25 in cultured media of Caco-2 cells treated with Baf $\mathrm{A} 1(20 \mathrm{nM})$ and torin $1(2 \mu \mathrm{M})$ under nutrient deprivation for $6,12,24,36$, and 48 h. Activity was normalized to cell viability. (B) ELISA assay of IL-17E/25 of cultured media in rhlL-1 $\beta$-treated $(4,20$, and $40 \mathrm{ng} / \mathrm{ml})$ Caco-2 cells for 36 h. (C) Caco-2 cells were treated with rhlL-1 $\beta(4 \mathrm{ng} / \mathrm{ml})$ and rhlL-25 $(0.5 \mathrm{ng} / \mathrm{ml})$ for $36 \mathrm{~h}$, and subsequently immunofluorescent staining of E-cadherin (green fluorescence) and Vimentin (red fluorescence). The nuclei were visualized with DAPI (blue fluorescence). Scale bar: $125 \mu \mathrm{m}$. The number of vimentin-positive cells were quantified and presented as a graph (vimentin-positive cells per total cells); counts are the mean \pm SD of 3 independent experiments.

The cells treated with both rhlL-1 $\beta$ and rhlL-17E/25 showed low expression levels of E-cadherin with disrupted junction and a high expression level of vimentin. Taken together, these results suggest that the secretion of $\mathrm{IL}-17 \mathrm{E} / 25$, which is promoted by the activation of IL-1 $\beta$, diminishes the function of the intestinal epithelial barrier through an EMT increase.

In conclusion, we demonstrated that autophagy down-regulated NLRP3 inflammasome activation and maintained the intestinal epithelial barrier by suppressing EMT under nutrient deprivation. Nutrient deprivation elevated NLRP3 inflammasomes in the early phase, after which the activated NLRP3 inflammasomes were gradually degraded by the ubiquitin-mediated autophagic process. If autophagy is impaired during nutrient deprivation, excessive NLRP3 inflammasomes are induced, leading to an enhanced EMT and intestinal permeability. Furthermore, IL-17E/25, which is secreted by IL-1 $\beta$, was found to be a candidate cytokine to invoke the starvation-induced loss of the intestinal epithelial barrier via NLRP3 inflammasome activation. Thus, our findings suggest that IL-17E/25 is a novel therapeutic target in inflammatory diseases of the gastrointestinal tract.

\section{MATERIALS AND METHODS}

Materials and Methods are available in the Supplementary Information.

\section{ACKNOWLEDGEMENTS}

This research was supported by grants from the National Research Foundation of Korea (2017R1E1A1A01074656) funded by the Korean government. We would like to thank Editage (www.editage.co.kr) for English language editing.

\section{CONFLICTS OF INTEREST}

The authors have no conflicting interests.

\section{REFERENCES}

1. Vaziri ND, Yuan J, Nazertehrani S, Ni Z and Liu S (2013) Chronic kidney disease causes disruption of gastric and small intestinal epithelial tight junction. Am J Nephrol 38, 99-103

2. Tabat MW, Marques TM, Markgren M, Lofvendahl L, Brummer RJ and Wall R (2020) Acute effects of butyrate on induced hyperpermeability and tight junction protein expression in human colonic tissues. Biomolecules 10, 766

3. Zareie M, Johnson-Henry K, Jury J et al (2006) Probiotics prevent bacterial translocation and improve intestinal barrier function in rats following chronic psychological stress. Gut 55, 1553-1560

4. Kiesslich R, Duckworth CA, Moussata D et al (2012) Local barrier dysfunction identified by confocal laser endomicroscopy predicts relapse in inflammatory bowel disease. Gut 61, 1146-1153

5. Bouma G, Oudkerk Pool M, Crusius JB et al (1997) Evidence for genetic heterogeneity in inflammatory bowel disease (IBD); HLA genes in the predisposition to suffer from ulcerative colitis (UC) and Crohn's disease (CD). Clin Exp Immunol 109, 175-179

6. Ukena SN, Singh A, Dringenberg U et al (2007) Probiotic Escherichia coli Nissle 1917 inhibits leaky gut by enhancing mucosal integrity. PLoS One 2, e1308

7. Kanak MA, Shahbazov R, Yoshimatsu G, Levy MF, Lawrence MC and Naziruddin B (2017) A small molecule inhibitor of NF kappa B blocks ER stress and the NLRP3 inflammasome and prevents progression of pancreatitis. J Gastroenterol 52, 352-365

8. Martinon F, Burns K and Tschopp J (2002) The inflammasome: a molecular platform triggering activation of inflammatory caspases and processing of prolL-beta. Mol Cell 10, 417-426

9. Wang H, Wang G, Liang Y et al (2019) Redox regulation of hepatic NLRP3 inflammasome activation and immune dysregulation in trichloroethene-mediated autoimmunity. Free Radic Biol Med 143, 223-231

10. Al-Sadi RM and Ma TY (2007) IL-1beta causes an increase in intestinal epithelial tight junction permeability. J Immunol 178, 4641-4649

11. Tang Z, Lin MG, Stowe TR et al (2013) Autophagy promotes primary ciliogenesis by removing OFD1 from centriolar satellites. Nature 502, 254-257

12. Pilli M, Arko-Mensah J, Ponpuak M et al (2012) TBK-1 promotes autophagy-mediated antimicrobial defense by con- 
trolling autophagosome maturation. Immunity 37, 223-234

13. Bruns C, MCCaffery JM, Curwin AJ, Duran JM and Malhotra V (2011) Biogenesis of a novel compartment for autophagosome-mediated unconventional protein secretion. J Cell Biol 195, 979-992

14. Houtman J, Freitag K, Gimber N, Schmoranzer J, Heppner FL and Jendrach M (2019) Beclin1-driven autophagy modulates the inflammatory response of microglia via NLRP3. EMBO J 38, e99430

15. Saitoh T, Fujita N, Jang MH et al (2008) Loss of the autophagy protein Atg16L1 enhances endotoxin-induced IL-1 beta production. Nature 456, 264-268

16. Wong M, Ganapathy AS, Suchanec E, Laidler L, Ma T and Nighot P (2019) Intestinal epithelial tight junction barrier regulation by autophagy-related protein ATG6/beclin 1 . Am J Physiol Cell Physiol 316, C753-C765

17. Zhong Z, Umemura A, Sanchez-Lopez E et al (2016) NFkappaB restricts inflammasome activation via elimination of damaged mitochondria. Cell 164, 896-910

18. lida T, Onodera K and Nakase H (2017) Role of autophagy in the pathogenesis of inflammatory bowel disease. World J Gastroenterol 23, 1944-1953

19. Ko JH, Yoon SO, Lee HJ and Oh JY (2017) Rapamycin regulates macrophage activation by inhibiting NLRP3 inflammasome-p38 MAPK-NFkappaB pathways in autophagyand p62-dependent manners. Oncotarget 8, 40817-40831

20. Yan Y, Jiang W, Liu L et al (2015) Dopamine controls systemic inflammation through inhibition of NLRP3 inflam- masome. Cell 160, 62-73

21. Chiu HW, Chen $\mathrm{CH}$, Chang JN, Chen $\mathrm{CH}$ and $\mathrm{Hsu} \mathrm{YH}$ (2016) Far-infrared promotes burn wound healing by suppressing NLRP3 inflammasome caused by enhanced autophagy. J Mol Med (Berl) 94, 809-819

22. Li Y, Zhang L, Zhou J et al (2015) Nedd4 E3 ubiquitin ligase promotes cell proliferation and autophagy. Cell Prolif 48, 338-347

23. Lopez-Castejon G (2020) Control of the inflammasome by the ubiquitin system. FEBS J 287, 11-26

24. Ruiz PA, Moron B, Becker HM et al (2017) Titanium dioxide nanoparticles exacerbate DSS-induced colitis: role of the NLRP3 inflammasome. Gut 66, 1216-1224

25. Zaki MH, Boyd KL, Vogel $P$, Kastan MB, Lamkanfi $M$ and Kanneganti TD (2010) The NLRP3 inflammasome protects against loss of epithelial integrity and mortality during experimental colitis. Immunity 32, 379-391

26. Rera M, Clark RI and Walker DW (2012) Intestinal barrier dysfunction links metabolic and inflammatory markers of aging to death in Drosophila. Proc Natl Acad Sci U S A 109, 21528-21533

27. Liu L, Guo X, Rao JN et al (2009) Polyamines regulate E-cadherin transcription through c-Myc modulating intestinal epithelial barrier function. Am J Physiol Cell Physiol 296, C801-810

28. Song $X, \mathrm{He} X, \mathrm{Li} X$ and Qian $Y$ (2016) The roles and functional mechanisms of interleukin-17 family cytokines in mucosal immunity. Cell Mol Immunol 13, 418-431 